\title{
A Bijection between Atomic Partitions and Unsplitable Partitions
}

\author{
William Y.C. Chen ${ }^{1}$, Teresa X.S. Li ${ }^{2}$ \\ ${ }^{1,2}$ Center for Combinatorics, LPMC-TJKLC \\ Nankai University, Tianjin 300071, P.R. China \\ ${ }^{1}$ chen@nankai.edu.cn, ${ }^{2}$ lxs@cfc.nankai.edu.cn \\ David G.L. Wang ${ }^{3}$ \\ ${ }^{3}$ Beijing International Center for Mathematical Research \\ Peking University, Beijing 100871, P.R. China \\ ${ }^{3}$ wgl@math.pku.edu.cn
}

Submitted: Sep 3, 2010; Accepted: Dec 23, 2010; Published: Jan 5, 2011

Mathematics Subject Classification: 05A18, 05A19, 05E05

\begin{abstract}
In the study of the algebra NCSym of symmetric functions in noncommutative variables, Bergeron and Zabrocki found a free generating set consisting of power sum symmetric functions indexed by atomic partitions. On the other hand, Bergeron, Reutenauer, Rosas, and Zabrocki studied another free generating set of NCSym consisting of monomial symmetric functions indexed by unsplitable partitions. Can and Sagan raised the question of finding a bijection between atomic partitions and unsplitable partitions. In this paper, we provide such a bijection.
\end{abstract}

\section{Introduction}

In their study of the algebra NCSym of symmetric functions in noncommutative variables, Rosas and Sagan [5] introduced a vector space with a basis

$$
\left\{p_{\pi} \mid \pi \text { is a set partition }\right\}
$$

where $p_{\pi}$ is the power sum symmetric function in noncommutative variables. Bergeron, Hohlweg, Rosas, and Zabrocki [1] obtained the following formula

$$
p_{\pi \mid \sigma}=p_{\pi} p_{\sigma}
$$

where $\pi \mid \sigma$ denotes the slash product of $\pi$ and $\sigma$. It follows that, as an algebra, NCSym is freely generated by $p_{\pi}$ with $\pi$ atomic, see Bergeron and Zabrocki [3]. It should be noted 
that Wolf [6] showed that NCSym is freely generated by another basis. A combinatorial characterization of the generating set of Wolf has been found by Bergeron, Reutenauer, Rosas, and Zabrocki [2]. More precisely, they introduced the notion of unsplitable partitions and proved that the generating set of Wolf can be described as the set of monomial symmetric functions in noncommutative variables indexed by unsplitable partitions.

Let $[n]$ denote the set $\{1,2, \ldots, n\}$. Taking the degree into account, one sees that the number of atomic partitions of $[n]$ equals the number of unsplitable partitions of $[n]$. Recently, Can and Sagan [4] raised the question of finding a combinatorial proof of this fact. The objective of this paper is to present such a proof.

\section{The bijection}

In this section we construct a bijection between the set of atomic partitions of $[n]$ and the set of unsplitable partitions of $[n]$.

Let us begin with an overview of terminology. Let $X$ be a finite set of positive integers. A partition $\pi$ of $X$ is a family $\left\{B_{1}, B_{2}, \ldots, B_{k}\right\}$ of disjoint nonempty subsets of $X$ whose union is $X$. The subsets $B_{i}$ are called blocks of $\pi$. Without loss of generality, we may assume that the blocks of a partition are arranged in the increasing order of their minimal elements, and that the elements in each block are written in increasing order.

Let $\pi$ be a partition of $X$ and $S \subseteq X$. We say that $\sigma$ is the restriction of $\pi$ on $S$, denoted by $\sigma=\pi_{S}$, if $\sigma$ is a partition of $S$ such that any two elements lie in the same block of $\sigma$ if and only if they are in the same block of $\pi$. In other words, $\pi_{S}$ is obtained from $\pi$ by removing all elements that do not belong to $S$. For two positive integers $i$ and $j$ with $i<j$, we use $[i, j]$ to denote the set $\{i, i+1, \ldots, j\}$. For example, if

$$
\pi=\{\{1,3,5,6\},\{2,7,9\},\{4,8,10\}\},
$$

then

$$
\pi_{[5,10]}=\{\{5,6\},\{7,9\},\{8,10\}\} .
$$

Let $\Pi_{n}$ be the set of partitions of $[n]$. Assume that

$$
\pi=\left\{B_{1}, B_{2}, \ldots, B_{k}\right\} \in \Pi_{m}, \quad \sigma=\left\{C_{1}, C_{2}, \ldots, C_{l}\right\} \in \Pi_{n} .
$$

The slash product of $\pi$ and $\sigma$, denoted by $\pi \mid \sigma$, is defined to be the partition obtained by joining the blocks of $\pi$ and the blocks of the partition

$$
\sigma+m=\left\{C_{1}+m, C_{2}+m, \ldots, C_{l}+m\right\}
$$

that is,

$$
\pi \mid \sigma=\left\{B_{1}, B_{2}, \ldots, B_{k}, C_{1}+m, C_{2}+m, \ldots, C_{l}+m\right\},
$$

where $C_{i}+m$ denotes the block obtained by adding $m$ to each element in $C_{i}$. It can be seen that $\pi \mid \sigma \in \Pi_{m+n}$. A partition $\pi$ is said to be atomic if there are no nonempty partitions $\sigma$ and $\tau$ such that $\pi=\sigma \mid \tau$. Let $\mathcal{A}_{n}$ be the set of atomic partitions of $[n]$. For example, for $n=3$ there are two atomic partitions $\{\{1,3\},\{2\}\}$ and $\{\{1,2,3\}\}$. 
The split product of $\pi$ and $\sigma$, denoted by $\pi \circ \sigma$, is given by

$$
\pi \circ \sigma= \begin{cases}\left\{B_{1} \cup\left(C_{1}+m\right), \ldots, B_{k} \cup\left(C_{k}+m\right), C_{k+1}+m, \ldots, C_{l}+m\right\}, & \text { if } k \leq l ; \\ \left\{B_{1} \cup\left(C_{1}+m\right), \ldots, B_{l} \cup\left(C_{l}+m\right), B_{l+1}, \ldots, B_{k}\right\}, & \text { if } k>l .\end{cases}
$$

Clearly, $\pi \circ \sigma \in \Pi_{m+n}$. A partition is said to be splitable if it is the split product of two nonempty partitions. Otherwise, it is said to be unsplitable. Denote by $\mathcal{U S}_{n}$ the set of unsplitable partitions of $[n]$. For example, for $n=3$ there are two unsplitable partitions $\{\{1\},\{2,3\}\}$ and $\{\{1\},\{2\},\{3\}\}$.

To describe our bijection, we first notice that it is possible for a partition to be atomic and unsplitable at the same time. For example, the partition

$$
\{\{1,3,7\},\{2,6\},\{4,5,8\}\}
$$

is both atomic and unsplitable. Our bijection will be concerned with atomic partitions that are splitable and unsplitable partitions that are not atomic. In other words, we shall establish a bijection

$$
\varphi: \mathcal{A}_{n} \backslash \mathcal{U} \mathcal{S}_{n} \longrightarrow \mathcal{U} \mathcal{S}_{n} \backslash \mathcal{A}_{n}
$$

For the sake of presentation, let us introduce a notation. Let $X=\left\{x_{1}, \ldots, x_{n}\right\}$ be a finite set of positive integers such that $x_{1}<\cdots<x_{n}$. Suppose that $\pi=\left\{B_{1}, B_{2}, \ldots, B_{k}\right\}$ is a partition of $X$. Let $r$ be the largest integer $j$ such that

$$
B_{j} \cup B_{j+1} \cup \cdots \cup B_{k}=\left\{x_{t}, x_{t+1}, \ldots, x_{n}\right\}
$$

for some $t$. The existence of such an integer $r$ is evident. We define

$$
R(\pi)=\left\{B_{r}, B_{r+1}, \ldots, B_{k}\right\} .
$$

Given the partition $\pi=\{\{1,3,5,6\},\{2,7,9\},\{4,8,10\}\}$ as in (2.1), we have

$$
R\left(\pi_{[5,10]}\right)=\{\{7,9\},\{8,10\}\} .
$$

In the above notation, we see that $\pi$ is atomic if and only if $\pi=R(\pi)$.

We are now ready to present the map $\varphi$. Suppose that $\pi=\left\{B_{1}, B_{2}, \ldots, B_{k}\right\} \in$ $\mathcal{A}_{n} \backslash \mathcal{U} \mathcal{S}_{n}$. It consists of three steps.

Step 1 . Let $i$ be the smallest element in $B_{1}$ such that $\pi=\pi_{[i-1]} \circ\left(\pi_{[i, n]}-i+1\right)$. The existence of the element $i$ is guaranteed by the condition that $\pi$ is splitable.

Step 2. Let $j$ be the smallest element in the underlying set of the partition $R\left(\pi_{[i, n]}\right)$. We see that $2 \leq i \leq j \leq n$ and $R\left(\pi_{[i, n]}\right)=\pi_{[j, n]}$.

Step 3. Set $\varphi(\pi)$ to be the partition $\pi_{[j-1]} \mid\left(\pi_{[j, n]}-j+1\right)$.

For example, considering the partition given in (2.1), we have $i=5$. By (2.3), we get $j=7$ and thus

$$
\varphi(\pi)=\{\{1,3,5,6\},\{2\},\{4\},\{7,9\},\{8,10\}\} .
$$

Theorem 2.1 The map $\varphi$ is a bijection from $\mathcal{A}_{n} \backslash \mathcal{U} \mathcal{S}_{n}$ to $\mathcal{U} \mathcal{S}_{n} \backslash \mathcal{A}_{n}$. 
Proof. First, we claim that $\varphi(\pi) \in \mathcal{U S}_{n} \backslash \mathcal{A}_{n}$. Since $2 \leq j \leq n$, both $\pi_{[j-1]}$ and $\pi_{[j, n]}$ are nonempty partitions. This implies that $\varphi(\pi) \notin \mathcal{A}_{n}$.

We next proceed to show that $\varphi(\pi)$ is unsplitable. To this end, let

$$
\pi_{[j-1]}=\left\{C_{1}, C_{2}, \ldots, C_{s}\right\}, \quad \pi_{[j, n]}=\left\{D_{1}, D_{2}, \ldots, D_{t}\right\} .
$$

Then

$$
\varphi(\pi)=\left\{C_{1}, C_{2}, \ldots, C_{s}, D_{1}, D_{2}, \ldots, D_{t}\right\} .
$$

Assume to the contrary that $\varphi(\pi)$ is splitable, namely, there exists an element $l \in C_{1}$ such that

$$
\varphi(\pi)=\varphi(\pi)_{[l-1]} \circ\left(\varphi(\pi)_{[l, n]}-l+1\right) .
$$

Since $n$ belongs to some block $D_{h}$, by the definition of the split product, we deduce that

$$
C_{p} \cap[l, n] \neq \emptyset, \quad \text { for each } 1 \leq p \leq s .
$$

By the choice of $i$, we find that $l \geq i$. Recall that $\pi=\left\{B_{1}, B_{2}, \ldots, B_{k}\right\}$. By the definition of $\pi_{[j, n]}$, we may assume that the block $D_{1}$ of $\pi_{[j, n]}$ is contained in some block $B_{r}$ of $\pi$. If $D_{1}=B_{r}$, then the smallest element of $B_{r}$ is $j$. Therefore all elements in $B_{r+1}, B_{r+2}, \ldots, B_{k}$ are larger than $j$. Now, by the choice of $j$, we deduce that

$$
B_{r} \cup B_{r+1} \cup \cdots \cup B_{k}=[j, n] .
$$

Consequently,

$$
\pi=\pi_{[j-1]} \mid\left(\pi_{[j, n]}-j+1\right)
$$

which contradicts the assumption that $\pi$ is atomic. Hence we have $D_{1} \neq B_{r}$, and so $C_{r}=B_{r} \backslash D_{1} \neq \emptyset$. Since $D_{1}$ is a block of the partition $\pi_{[i, n]}$, it consists of all the elements in $B_{r}$ that are larger than or equal to $i$. In other words, each element in $C_{r}$ is less than $i$. This yields that $C_{r} \cap[l, n]=\emptyset$, a contradiction to (2.5). Thus we have proved the claim that $\varphi(\pi) \in \mathcal{U} \mathcal{S}_{n} \backslash \mathcal{A}_{n}$.

We now define a map

$$
\psi: \mathcal{U S}_{n} \backslash \mathcal{A}_{n} \longrightarrow \mathcal{A}_{n} \backslash \mathcal{U} \mathcal{S}_{n}
$$

and we shall show that $\psi$ is the inverse of $\varphi$. Let $\sigma=\left\{B_{1}, B_{2}, \ldots, B_{k}\right\} \in \mathcal{U} \mathcal{S}_{n} \backslash \mathcal{A}_{n}$. Step 1. Let $j$ be the smallest element in the underlying set of the partition $R(\sigma)$. Step 2. If $\sigma_{[j-1]}$ is unsplitable, then set

$$
\psi(\sigma)=\sigma_{[j-1]} \circ\left(\sigma_{[j, n]}-j+1\right) .
$$

If $\sigma_{[j-1]}$ is splitable, then choose $i$ to be the smallest element in $B_{1}$ such that

$$
\sigma_{[j-1]}=\sigma_{[i-1]} \circ\left(\sigma_{[i, j-1]}-i+1\right) .
$$

Let $q=\min \left\{l \mid B_{l} \subseteq[i-1]\right\}$, and let $B_{r}$ be the first block in the partition $R(\sigma)$. If $2 r-q-1 \leq k$, then set

$$
\psi(\sigma)=\left\{B_{1}, \ldots, B_{q-1}, B_{q} \cup B_{r}, \ldots, B_{r-1} \cup B_{2 r-q-1}, B_{2 r-q}, \ldots, B_{k}\right\} .
$$


If $2 r-q-1>k$, then set

$$
\psi(\sigma)=\left\{B_{1}, \ldots, B_{q-1}, B_{q} \cup B_{r}, \ldots, B_{q+k-r} \cup B_{k}, B_{q+k-r+1}, \ldots, B_{r-1}\right\} .
$$

First, we show that $\psi$ is well-defined. For any $\sigma \in \mathcal{U} \mathcal{S}_{n} \backslash \mathcal{A}_{n}$, we notice that in Step 1 of the above construction of $\psi$, the element $j$ always exists. Moreover, we observe that $j \geq 2$ since $\sigma$ is not atomic. By the choice of $j$, we have

$$
\begin{aligned}
\sigma_{[j-1]} & =\left\{B_{1}, B_{2}, \ldots, B_{r-1}\right\}, \\
\sigma_{[j, n]} & =R(\sigma)=\left\{B_{r}, B_{r+1}, \ldots, B_{k}\right\} .
\end{aligned}
$$

Since $\sigma$ is unsplitable, we can always find the element $q$. Otherwise, if every block $B_{1}, B_{2}, \ldots, B_{k}$ contains an element in $[i, n]$, by the assumption $(2.6)$, we have $B_{p} \cap[i, n] \neq \emptyset$ for any $1 \leq p \leq k$, and

$$
\min \left(B_{1} \cap[i, n]\right)<\min \left(B_{2} \cap[i, n]\right)<\cdots<\min \left(B_{k} \cap[i, n]\right) .
$$

This implies that

$$
\sigma=\sigma_{[i-1]} \circ\left(\sigma_{[i, n]}-i+1\right),
$$

a contradiction to the fact that $\sigma$ is unsplitable. This confirms the existence of the element $q$. At this point, we still need to show that $\psi(\sigma) \in \mathcal{A}_{n} \backslash \mathcal{U} \mathcal{S}_{n}$. It is clear from the above construction that $\psi(\sigma)$ is splitable. For the case when $\sigma_{[j-1]}$ is unsplitable, it is easily seen that $\psi(\sigma)$ is atomic. When $\sigma_{[j-1]}$ is splitable, since $i \in B_{1}$ and $B_{q} \subseteq[i-1]$, we find that $\psi(\sigma)$ is atomic. Thus we have shown that $\psi(\sigma) \in \mathcal{A}_{n} \backslash \mathcal{U} \mathcal{S}_{n}$. Consequently, $\psi$ is well-defined.

It remains to show that $\psi$ is indeed the inverse of $\varphi$, that is, $\psi(\varphi(\pi))=\pi$ for any $\pi \in \mathcal{A}_{n} \backslash \mathcal{U S}_{n}$. As in the construction of $\varphi$, we assume that $i$ is the smallest element in the first block of $\pi$ such that

$$
\pi=\pi_{[i-1]} \circ\left(\pi_{[i, n]}-i+1\right)
$$

and $j$ is the smallest element in the underlying set of the partition $R\left(\pi_{[i, n]}\right)$. First we notice that the element $j$ chosen during the process of computing $\varphi(\pi)$ coincides with the element $j$ defined when computing $\psi(\varphi(\pi))$, since

$$
R(\varphi(\pi))=\varphi(\pi)_{[j, n]}=\pi_{[j, n]},
$$

by the definition of $\varphi$. Moreover, from Step 3 in the construction of $\varphi$, we see that

$$
\varphi(\pi)_{[j-1]}=\pi_{[j-1]}
$$

Now we need to consider two cases. If $R\left(\pi_{[i, n]}\right)=\pi_{[i, n]}$, i.e., $i=j$, then (2.8) implies that $\varphi(\pi)_{[j-1]}=\pi_{[i-1]}$. Since $\pi_{[i-1]}$ is unsplitable by the choice of $i$, from (2.7) it follows that

$$
\psi(\varphi(\pi))=\varphi(\pi)_{[j-1]} \circ\left(\varphi(\pi)_{[j, n]}-j+1\right)=\pi_{[i-1]} \circ\left(\pi_{[i, n]}-i+1\right)=\pi .
$$


If $R\left(\pi_{[i, n]}\right) \neq \pi_{[i, n]}$, that is, $i<j$, then we have

$$
\varphi(\pi)_{[j-1]}=\pi_{[j-1]}=\pi_{[i-1]} \circ\left(\pi_{[i, j-1]}-i+1\right) .
$$

This implies that $\varphi(\pi)_{[j-1]}$ is splitable. Recall that if the first block $D_{1}$ of $\pi_{[j, n]}$ is contained in the block $B_{r}$ of $\pi$, then $C_{r}=B_{r} \backslash D_{1}$ is a block of $\pi_{[j-1]}$ which consists of elements that are smaller than $i$. So we may assume that

$$
\varphi(\pi)_{[j-1]}=\left\{C_{1}^{\prime} \cup C_{1}^{\prime \prime}, C_{2}^{\prime} \cup C_{2}^{\prime \prime}, \ldots, C_{u-1}^{\prime} \cup C_{u-1}^{\prime \prime}, C_{u}, \ldots, C_{s}\right\},
$$

where

$$
\pi_{[i-1]}=\left\{C_{1}^{\prime}, C_{1}^{\prime}, \ldots, C_{u-1}^{\prime}, C_{u}, \ldots, C_{s}\right\}
$$

for some $u \leq s$ and

$$
\pi_{[i, j-1]}=\left\{C_{1}^{\prime \prime}, C_{2}^{\prime \prime}, \ldots, C_{u-1}^{\prime \prime}\right\} .
$$

Since $\pi=\pi_{[i-1]} \circ\left(\pi_{[i, n]}-i+1\right)$ and $\pi_{[i, n]}=\pi_{[i, j-1]} \cup \pi_{[j, n]}$, we deduce that $u=r$ and

$$
\pi=\left\{C_{1}^{\prime} \cup C_{1}^{\prime \prime}, \ldots, C_{r-1}^{\prime} \cup C_{r-1}^{\prime \prime}, C_{r} \cup D_{1}, C_{r+1} \cup D_{2}, \ldots\right\},
$$

where $\pi_{[j, n]}=\left\{D_{1}, D_{2}, \ldots, D_{t}\right\}$, and the last block of $\pi$ depends on whether $s-r+1 \leq t$ or $s-r+1>t$. In other words, $\pi$ can be recovered from $\varphi(\pi)$ as the following procedure. First, we combine the first block $D_{1}$ of $\varphi(\pi)_{[j, n]}$ with the block $C_{r}$ of $\varphi(\pi)_{[j-1]}$. Then we combine the second block of $\varphi(\pi)_{[j, n]}$ with $C_{r+1}$, and so on. This process coincides exactly with the construction of $\psi(\varphi(\pi))$ when $\varphi(\pi)_{[j-1]}$ is splitable. Thus we deduce that $\psi(\varphi(\pi))=\pi$. This completes the proof.

We conclude with some examples to illustrate the maps $\varphi$ and $\psi$. Assume that

$$
\sigma=\{\{1,3,5,6\},\{2\},\{4\},\{7,9\},\{8,10\}\}
$$

which is the partition given in (2.4). It can be checked that $\psi(\sigma)=\pi$ as given in (2.1). In fact,

$$
R(\sigma)=\{\{7,9\},\{8,10\}\}
$$

and thus $j=7$ is the smallest element in the underlying set of $R(\sigma)$. Now,

$$
\sigma_{[j-1]}=\left\{B_{1}, B_{2}, B_{3}\right\}
$$

is splitable, where $B_{1}=\{1,3,5,6\}, B_{2}=\{2\}$, and $B_{3}=\{4\}$. In Step 2 of the map $\psi$, $i=5$ is the smallest element in $B_{1}$ such that

$$
\sigma_{[j-1]}=\sigma_{[i-1]} \circ\left(\sigma_{[i, j-1]}-i+1\right) .
$$

Since $B_{2}$ is the first block of $\sigma_{[j-1]}$ that is contained in $[i-1]$, we get $\psi(\sigma)=\pi$.

Below is an example for the case when $\varphi(\pi)_{[j-1]}$ is unsplitable. Let

$$
\pi=\{\{1,3,5,8\},\{2,6,9\},\{4,7,10\}\} .
$$


In Step 1 of the map $\varphi$, we have $i=5$ and

$$
\pi_{[5,10]}=\{\{5,8\},\{6,9\},\{7,10\}\} .
$$

Since $R\left(\pi_{[5,10]}\right)=\pi_{[5,10]}$, we see that $j=5=i$, and so

$$
\varphi(\pi)=\{\{1,3\},\{2\},\{4\},\{5,8\},\{6,9\},\{7,10\}\} .
$$

Conversely, let $\sigma$ be the partition given in (2.10). It is easy to verify that the partition $R(\sigma)$ agrees with the partition given in (2.9). So we get $j=5$ and

$$
\sigma_{[j-1]}=\{\{1,3\},\{2\},\{4\}\}
$$

which is unsplitable. So we arrive at $\psi(\sigma)=\pi$.

Acknowledgments. We wish to thank the referee for helpful suggestions. This work was supported by the 973 Project, the PCSIRT Project of the Ministry of Science and Technology, and the National Science Foundation of China.

\section{References}

[1] N. Bergeron, C. Hohlweg, M.H. Rosas and M. Zabrocki, Grothendieck bialgebras, partition lattices, and symmetric functions in noncommutative variables, Electron. J. Combin. 13, 1 (2006), \#R75.

[2] N. Bergeron, C. Reutenauer, M.H. Rosas and M. Zabrocki, Invariants and coinvariants of the symmetric group in noncommuting variables, Canad. J. Math. 60, 2 (2008), 266-296.

[3] N. Bergeron and M. Zabrocki, The Hopf algebras of symmetric functions and quasisymmetric functions in non-commutative variables are free and cofree, arXiv:math.CO/0509265.

[4] M.B. Can and B.E. Sagan, Partitions, rooks, and symmetric functions in noncommuting variables, arXiv:math.CO/1008.2950.

[5] M.H. Rosas and B.E. Sagan, Symmetric functions in noncommuting variables, Trans. Amer. Math. Soc. 358, 1 (2006), 215-232.

[6] M.C. Wolf, Symmetric functions of non-commutative elements, Duke Math. J. 2 (1936), 626-637. 